\title{
Quasi-Linear Transformations, Numeration Systems and Fractals ${ }^{\star}$
}

\author{
Marie-Andrée Jacob-Da Col and Pierre Tellier \\ LSIIT-UMR 7005, Pôle API, Bd Sébastien Brant \\ 67412 Illkirch Cedex France \\ \{dacolm,tellier\}@unistra.fr
}

\begin{abstract}
In this paper we will define relations between quasi-linear transformations, numeration systems and fractals. A Quasi-Linear Transformation (QLT) is a transformation on $\mathbb{Z}^{n}$ which corresponds to the composition of a linear transformation with an integer part function. We will first give some theoretical results about QLTs. We will then point out relations between QLTs, numeration systems and fractals. These relations allow us to define new numeration systems, fractals associated with them and n-dimensional fractals. With help of some properties of the QLTs we can give the fractal dimension of these fractals.
\end{abstract}

Keywords: Gaussian integers, algebraic integers, numeration systems, discrete linear transformations, fractals.

\section{Introduction}

Fractal tiles generated by numeration systems and substitutions have been widely studied, see for example [1], [2, 5]. In this paper we define discrete linear transformations called QLT which generate tilings of $\mathbb{Z}^{n}$. The tilings studied here are self-similar, they allow us to generate n-dimensional fractals. In dimension two we point out relations between QLTs and numeration systems which allow us to define new numeration systems. These discrete linear transformations are also in relation with discrete lines [17, 13 .

Let $g$ be a linear transformation from $\mathbb{Z}^{n}$ to $\mathbb{Q}^{n}$, defined by a matrix $\frac{1}{w} A$ where $A$ is an integer matrix and $w$ a positive integer. If we compose this transformation with an integer part function we obtain a Quasi-Linear Transformation (QLT) from $\mathbb{Z}^{n}$ to $\mathbb{Z}^{n}$. We will only consider the integer part function with a positive remainder. We will denote it \lfloor\rfloor . If $x$ and $y$ are two integers, $\left\lfloor\frac{x}{y}\right\rfloor$ denotes the quotient of the Euclidean division of $x$ by $y$. We denote $G$ as the QLT defined by $g$. Main research results on QLTs are listed in the first section of this paper.

Let us consider $\beta$ an algebraic number and $\mathcal{D}$ a set of elements of $\mathbb{Z}[\beta]$. $\beta$ is a valid base using the digit set $\mathcal{D}$ if every integer $c \in \mathbb{Z}[\beta]$ has a unique representation (decomposition) of the form: $c=\sum_{j=0}^{n} a_{j} \beta^{j}$, where $a_{j} \in \mathcal{D}$ and

\footnotetext{
* This work was supported by the Agence Nationale de la Recherche through contract ANR-2010-BLAN-0205-01.
} 
$n \in \mathbb{N}$. Then $(\beta, \mathcal{D})$ is called a numeration system. The third section will point out some relations between QLTs and numeration systems when $\beta$ is a Gaussian integer $(\beta=a+\imath b$, with $a, b \in \mathbb{Z})$ or an algebraic integer of order $2\left(\beta^{2}+b \beta+a=0\right.$ with $a, b \in \mathbb{Z}$ ). These relations and some properties of QLTs will allow us to generate new numeration systems.

In the fourth section we will see how QLTs generate fractals (some of them are in relation with numeration systems). In $2 \mathrm{D}$ the fractal dimension of the border of these fractals can be determined by substitutions associated with the QLT. These substitutions have been defined in 8 and [11. For the n-dimensional fractals the fractal dimension is determined directely using properties of QLTs.

\section{Quasi-Linear Transformations}

In this section we recall definitions and results that can be found in [7], 10], 3], 4 and that are useful in the rest of the paper. The proposition 1 can be found in [10]. Proposition 2 extends to $\mathbb{Z}^{n}$ a result given in [7] and [10] in $\mathbb{Z}^{2}$.

Definition 1. Let $g$ be a linear transformation from $\mathbb{Z}^{n}$ to $\mathbb{Q}^{n}$, defined by a matrix $\frac{1}{w} A$ where $A=\left(a_{i, j}\right)_{1 \leq i, j \leq n}$ is an integer matrix and $w$ a positive integer. The Quasi-Linear Transformation or QLT associated with $g$ is the transformation of the discrete plane $\mathbb{Z}^{n}$ defined by the composition of $g$ with the greatest integer part function noted \lfloor\rfloor . This QLT is then noted $G$.

In the following we will $\operatorname{denote} \delta=\operatorname{det}(A)$, where $\operatorname{det}(A)$ is the $\operatorname{determinant}$ of $A$, and we'll assume that $\delta>0$. The linear transformation defined by the matrix $\frac{1}{\omega} A$ extends to $\mathbb{R}^{n}$ and for each point $Y$ of $\mathbb{R}^{n}$, there exists a unique point $X$ of $\mathbb{R}^{n}$ such that $Y=g(X)$. This is not the same for a QLT. Indeed, each point of $\mathbb{Z}^{n}$ can have either none, one or several antecedents: The set of antecedents of $X \in \mathbb{Z}^{n}$ is then called tile with index $X$.

Definition 2. We call tile with index $X \in \mathbb{Z}^{n}$ and denote $P_{G, X}$ the set:

$$
P_{G, X}=\left\{Y \in \mathbb{Z}^{n} \mid G(Y)=X\right\}
$$

Definition 3. We call $p$-tile or tile of order $p \in \mathbb{N}$, with index $X \in \mathbb{Z}^{n}$, and denote $P_{X}^{p}$ the set: $P_{G, X}^{p}=\left\{Y \in \mathbb{Z}^{n} \mid G^{p}(Y)=X\right\}$ where $G^{p}$ denotes the transformation $G$ iterated $p$ times.

Definition 4. Two tiles are geometrically identical if one is the image of the other by a translation of an integer vector.

In this paper we focus on a particular type of QLTs, called "m-determinantal QLTs" and which are defined by $\frac{1}{w} A$ such that $\omega$ is a multiple of the determinant of $A$.

Definition 5. A QLT defined by a matrix $\frac{1}{w} A$ such that $w=m \operatorname{det}(A)$ where $m$ is a positive integer, is called a m-determinantal QLT. A 1-determinantal QLT will be called a determinantal QLT. 
In the following $G$ will denote a m-determinantal QLT associated with the matrix $\frac{1}{m \delta} A$ where $\delta=\operatorname{det}(A)$, and $g$ the associated rational linear transformation. To simplify the notations, tiles $P_{G, X}$ and $P_{G, X}^{p}$ will be noted $P_{X}$ and $P_{X}^{p}$. Moreover $(0,0, \ldots, 0) \in \mathbb{Z}^{n}$ will be simply noted $O$. Let denote $\widehat{A}^{T}$ the transpose the cofactor matrix of $A$, we have $A^{-1}=\frac{1}{\delta} \widehat{A}^{T}$. The following proposition allow to determine recursively the p-tiles of a m-determinantal QLT using translations and $P_{O}$, the proof can be found in [10].

Proposition 1. The p-tiles generated by a m-determinantal QLT are all geometrically identical and can be generated recursively by translations of $P_{O}$. More precisely, if $\mathcal{T}_{v}$ refers to the translation of the vector $v$ and if $\widehat{A}^{T}$ is the transpose of the cofactor matrix of $A$ we have, for all $p \geq 1$ :

$$
\begin{aligned}
P_{Y}^{p} & =\mathcal{T}_{\left(m \widehat{A}^{T}\right)^{p} Y} P_{O}^{p} \\
\text { and } \quad P_{O}^{p+1} & =\bigcup_{X \in P_{O}} \mathcal{T}_{\left(m \widehat{A}^{T}\right)^{p} X} P_{O}^{p}=\bigcup_{X \in P_{O}^{p}} \mathcal{T}_{\left(m \widehat{A}^{T}\right) X} P_{O}
\end{aligned}
$$

Example 1. Figure 1 shows the tile of order 2 of the QLT defined by $\frac{1}{8}\left(\begin{array}{rr}2 & 3 \\ -2 & 1\end{array}\right)$, this tile is composed of 8 tiles of order 1 . For each tile of order one we give the index of the tile which is a point of $P_{O}$. In figure 2 we see a fractal associated with this QLT. Figure 3 shows the tile of order 12 of the QLT defined by $\frac{1}{2}\left(\begin{array}{ll}-1 & 1 \\ -1 & -1\end{array}\right)$ and figure 4 shows the tile of order 7 of the QLT defined by $\frac{1}{2}\left(\begin{array}{ll}0 & -1 \\ 1 & -1\end{array}\right)$.

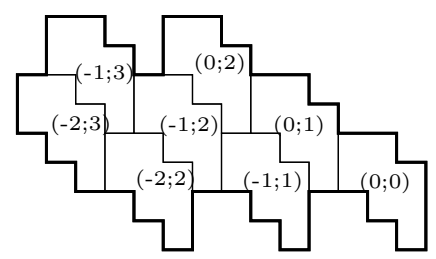

Fig. 1. Tile of order 2 containing 8 tiles of order 1

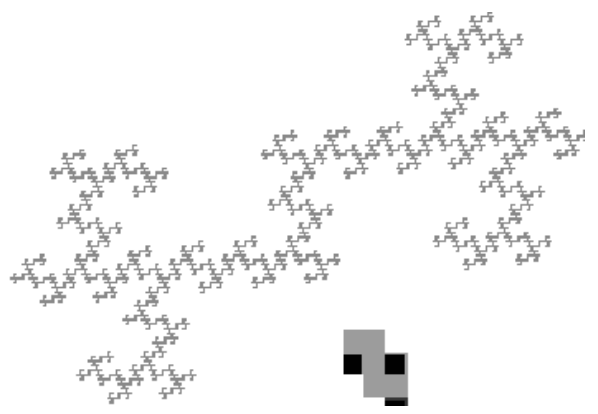

Fig. 2. Fractal associated with QLT

It is well known that if $g$ is a contracting linear transformation of $\mathbb{R}^{n}$ then $g$ has the origin as unique fixed point and for each $X \in \mathbb{R}^{n}$ the sequence $g^{n}(X)$ tends toward this fixed point. We will say that a QLT $G$ is contracting if $g$ is contracting. But such a QLT has not necessarily a unique fixed point. The 
behaviour under iteration of 2D contracting QLTs has been studied in [7, [16], [14] and [15. The following definition and theorem will be used to define new numeration systems.

Definition 6. A consistent Quasi-Linear Transformation is a QLT which has the origin as unique fixed point such that for each discrete point $Y$ the sequence $\left(G^{n}(Y)\right)_{n \geq 0}$ tends toward this unique fixed point.

Consider a 2D-QLT defined by $\frac{1}{\omega}\left(\begin{array}{ll}a & b \\ c & d\end{array}\right)$, the infinite norm of $g$ is then

$$
\|g\|_{\infty}=\frac{1}{\omega} \max (|a|+|b|,|c|+|d|) .
$$

The following theorem, proved in [7, states conditions such that a 2D-QLT is a consistent QLT.

Theorem 1. Let $G$ be a $Q L T$ in $\mathbb{Z}^{2}$ such that $\|g\|_{\infty}<1, G$ is a consistent $Q L T$ if and only if one of the three following conditions is verified:

$$
\begin{array}{llrl}
\text { (1) } b & \leq 0, a+b & \leq 0, c>0 \text { and } \quad d & \leq 0 \\
\text { (2) } a \leq 0, & b>0, c \leq 0 \text { and } c+d & \leq 0 \\
\text { (3) } a \leq 0, & b & \leq 0, c \leq 0 \text { and } \quad d & \leq 0
\end{array}
$$

Remark 1. It would be to long to give here the conditions such that a QLT of norm 1 is consistent. These conditions can be found in [7] and [1] and will be used to define some numeration systems.

The following proposition determines the number of points of a tile of order $p$ and will be used in the last section to determine the fractal dimension of n-dimensional fractals.

Proposition 2. The number of points of a p-tile generated by a m-determinantal $Q L T$ in $\mathbb{Z}^{n}$ is equal to $\delta^{p(n-1)} m^{n p}$.

Proof. Let first proof that the tile $P_{O}$ contains exactely $\delta^{n-1} m^{n}$ points.

Case of $m=1$ and $n=2$. It is known (see [7] and [15]) that in this case the number of points of $P_{O}$ equals $\delta$.

Case of $m=1$ and $n>2$. It has been proved in [7] and [3] that for each integer matrix $A$ it exists an unimodular matrix $U$ such that $A U=B$ where $B$ is an upper triangular integer matrix and that the points of the tiles of $A$ are in one-to-one correspondance with the points of the tiles of $B$. Therefore we will only do the proof for an upper triangular integer matrix $B=$ $\left(b_{i, j}\right)_{1 \leq i, j \leq n}$, we then have $\delta=b_{1,1} b_{2,2} \ldots b_{n, n}$. Let denote $P_{O}$, the tile associated with $B$ and $P_{X}^{\prime}$ the tiles associated with $B^{\prime}=\left(b_{i, j}^{\prime}\right)_{1 \leq i, j \leq n-1}$ with $b_{i, j}^{\prime}=b_{i+1, j+1}$ and $\delta^{\prime}=b_{2,2} b_{3,3} \ldots b_{n, n}=\operatorname{det}(B)$. By recurrence hypothesis we will suppose that the number of points of $P_{X}^{\prime}$ equals $\delta^{\prime n-2}$. We have: 


$$
\begin{aligned}
& \left(x_{1}, x_{2}, \ldots, x_{n}\right) \in P_{O}
\end{aligned}
$$

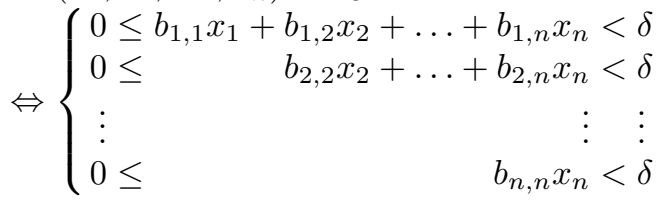

$$
\begin{aligned}
& \Leftrightarrow\left\{\begin{array}{rlrl}
-\frac{b_{1,2} x_{2}+\ldots+b_{1, n} x_{n}}{b_{1,1}} & \leq & x_{1} & <\delta^{\prime}-\frac{b_{1,2} x_{2}+\ldots+b_{1, n} x_{n}}{b_{1,1}} \\
0 & \leq \frac{b_{2,2} x_{2}+\ldots+b_{2, n} x_{n}}{\delta^{\prime}} & <b_{1,1} \\
\vdots & \vdots & \vdots \\
0 & \leq \frac{b_{n, n} x_{n}}{\delta^{\prime}} & <b_{1,1}
\end{array}\right. \\
& \Leftrightarrow\left\{\begin{array}{l}
-\frac{b_{1,2} x_{2}+\ldots+b_{1, n} x_{n}}{b_{1,1}} \leq x_{1}<\delta^{\prime}-\frac{b_{1,2} x_{2}+\ldots+b_{1, n} x_{n}}{b_{1,1}} \\
\left(x_{2}, x_{3}, \ldots, x_{n}\right) \in P_{i_{2}, i_{3}, \ldots, i_{n}}^{\prime} \text { with } i_{k}=0,1, \ldots, b_{1,1} \text { for } k=2,3, \ldots, n
\end{array}\right.
\end{aligned}
$$

The number of solutions for $x_{1}$ equals $\delta^{\prime}$ and each tile $P_{i_{2}, i_{3}, \ldots, i_{n}}^{\prime}$ contains $\delta^{\prime n-2}$, therefore we have $\delta^{\prime} \delta^{\prime n-2} b_{1,1}^{n-1}=\delta^{n-1}$ points.

If $m>1$, let denote $P_{X}^{\prime}$ the tiles associated with $\frac{1}{\delta} A$ and $P_{O}$ the tile associated with $\frac{1}{m \delta} A$, we have:

$$
\begin{aligned}
& \left(x_{1}, x_{2}, \ldots, x_{n}\right) \in P_{O} \\
\Leftrightarrow & \left\{\begin{array}{c}
0 \leq a_{1,1} x_{1}+a_{1,2} x_{2}+\ldots+a_{1, n} x_{n}<m \delta \\
\vdots \\
\vdots \\
0 \leq a_{n, 1} x_{1}+a_{n, 2} x_{2}+\ldots+a_{n, n} x_{n}<m \delta
\end{array}\right. \\
\Leftrightarrow & \left\{\begin{array}{cc}
0 \leq \frac{a_{1,1} x_{1}+a_{1,2} x_{2}+\ldots+a_{1, n} x_{n}}{\delta}<m \\
\vdots \\
\vdots \\
0 \leq \frac{a_{n, 1} x_{1}+a_{n, 2} x_{2}+\ldots+a_{n, n} x_{n}}{\delta}<m
\end{array}\right. \\
\Leftrightarrow & \left(x_{1}, x_{2}, \ldots, x_{n}\right) \in P_{i_{1}, i_{2}, \ldots, i_{n}}^{\prime} \text { with } i_{1}, i_{2}, \ldots, i_{n}=0,1, \ldots, m-1
\end{aligned}
$$

But each $P_{i_{1}, i_{2}, \ldots, i_{n}}^{\prime}$ contains $\delta^{n-1}$ points, it follows thats $P_{O}$ contains $\delta^{n-1} m^{n}$ points.

We conclude that the number of points of $P_{O}$ equals $\delta^{n-1} m^{n}$.

Let now supose that the number of points of $P_{O}^{p}$ equals $\delta^{p(n-1)} m^{n p}$, we have $P_{O}^{p+1}=\bigcup_{X \in P_{O}} \mathcal{T}_{\left(m \widehat{A}^{T}\right)^{p}{ }_{X} P_{O}^{p}}$ (see proposition 1), it follows that the number of points of $P_{O}^{p+1}$ equals $\delta^{p(n-1)} m^{n p} \delta^{n-1} m^{n}=\delta^{(p+1)(n-1)} m^{n(p+1)}$.

\section{Quasi-Linear Transformations and Numeration Systems}

Let $\beta$ denote a complex number and $\mathcal{D}$ a finite set of elements of $\mathbb{Z}[\beta]$. $(\beta, \mathcal{D})$ is a valid base for $\mathbb{Z}[\beta]$ if each element $c$ of $\mathbb{Z}[\beta]$ can be written uniquely in the form $c=c_{0}+c_{1} \beta+c_{2} \beta^{2} \ldots+c_{n} \beta^{n}$ with $c_{i} \in \mathcal{D}$ and $n \in \mathbb{N}$; the length of the decomposition is then $n+1$. We also say that $(\beta, \mathcal{D})$ is a numeration system of $\mathbb{Z}[\beta]$ and $\mathcal{D}$ is the set of digits of this numeration system. 


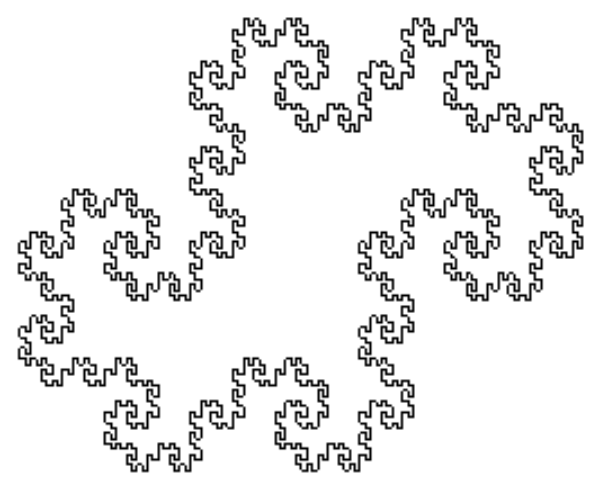

Fig. 3. Border of $P_{O}^{12}$

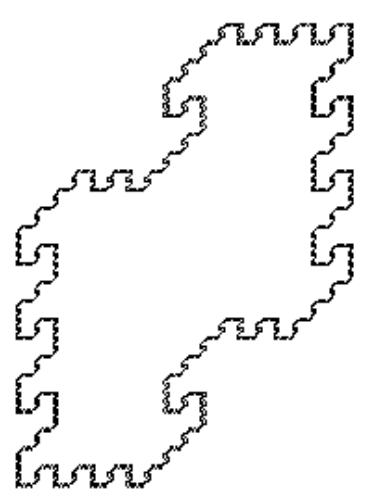

Fig. 4. Border of $P_{O}^{7}$

\subsection{Case of Gaussian Integers}

The results below can be found in [8]: They allow us to determine numeration systems of $\mathbb{Z}[i]$ by considering $\beta=a+i b$ a Gaussian integer and $\mathcal{D}$ a set of Gaussian integers.

Definition 7. Let $c=x+i y$ and $\beta=a+i b$ be two Gaussian integers. The integer division of $c$ by $\beta$, noted $\left\lfloor\frac{c}{\beta}\right\rfloor$, is defined by: $\left\lfloor\frac{c}{\beta}\right\rfloor=\left\lfloor\frac{a x+b y}{a^{2}+b^{2}}\right\rfloor+$ $i\left\lfloor\frac{-b x+a y}{a^{2}+b^{2}}\right\rfloor$.

This division corresponds to the usual division of complex numbers composed with the integer part function, so we have the following relation with QLTs.

Proposition 3. Let $\beta=a+i b$ and $c=x+i y$ be two Gaussian integers and let $c^{\prime}=x^{\prime}+i y^{\prime}=\left\lfloor\frac{c}{\beta}\right\rfloor$. The point $\left(x^{\prime}, y^{\prime}\right)$ is then the image of the point $(x, y)$ by the $Q L T G_{\beta}$ defined on $\mathbb{Z}^{2}$ by the matrix $\frac{1}{a^{2}+b^{2}}\left(\begin{array}{cc}a & b \\ -b & a\end{array}\right)$.

The following theorem and its proof can be found in [7] and [8].

Theorem 2. Let $\beta=a+i b$ be a Gaussian integer and $\mathcal{D}$ the set of Gaussian integers $c$ such that $\left\lfloor\frac{c}{\beta}\right\rfloor=0$, the three following properties are then equivalent:

1. $(\beta, \mathcal{D})$ is a numeration system,

2. The $Q L T G_{\beta}$ is a consistent Quasi-Linear Transformation,

3. $a \leq 0$ and $|a|+|b|>1$.

Remark 2. Consider the QLT $G_{\beta}$ and note $P_{O}$ and $P_{O}^{p}$ the tiles defined by this QLT. In order to determine the set of digits, we have to determine $P_{O}$. In 7 ] 
and [9] we can find an algorithm that allows to determine $P_{O} \cdot P_{O}^{p}$ represents the elements of $\mathbb{Z}[\beta]$ such that the decomposition in the numeration system is of length $p$. For example, figure 3 represents the elements of $\mathbb{Z}[-1+i]$ such that the decomposition in the numeration system $(-1+i,\{0,-1\})$ is of length 12 .

\subsection{Case of Algebraic Integers of Order 2}

Now we consider $\beta$ an algebraic integer such that $\beta^{2}+b \beta+a=0$ with $a, b \in \mathbb{Z}$. We only consider the case where $\beta$ is a complex number, that is to say $b^{2}-4 a<0$. We will define numeration systems of $\mathbb{Z}[\beta]$ where the digits are elements of $\mathbb{Z}[\beta]$. First we will define an integer division by $\beta$, this integer division corresponds to a QLT that will define the digits of the numeration system. We will define the division by using another base of $\mathbb{Z}[\beta]$, the QLT associated with the division will depend on these base, a good choice of the base will raise to a consistent QLT and so define a numeration system.

Remark 3. Let $\beta_{1}=q+\beta$ with $q \in \mathbb{Z}$, we have $\mathbb{Z}[\beta]=\mathbb{Z}\left[\beta_{1}\right]$, indeed:

$$
\begin{aligned}
& x=x_{1}+x_{2} \beta \in \mathbb{Z}[\beta] \\
\Leftrightarrow & \exists x_{1}, x_{2} \in \mathbb{Z} \mid x=x_{1}+x_{2} \beta \\
\Leftrightarrow & \exists x_{1}, x_{2} \in \mathbb{Z} \mid x=x_{1}-x_{2} q+x_{2} \beta_{1} \\
\Leftrightarrow & \exists x_{1}^{\prime}, x_{2}^{\prime} \in \mathbb{Z} \mid x=x_{1}^{\prime}+x_{2}^{\prime} \beta_{1} \\
\Leftrightarrow & x \in \mathbb{Z}\left[\beta_{1}\right]
\end{aligned}
$$

Let now define the integer division using this base. We have $\beta^{2}+b \beta+a=0$, so $\frac{1}{\beta}=-\frac{\beta+b}{a}=\frac{-\beta_{1}+q-b}{a}$ and $\beta_{1}^{2}=\beta_{1}(2 q-b)-q^{2}+q b-a$. Let $x=x_{1}+x_{2} \beta_{1}$, we have:

$$
\begin{aligned}
\frac{x}{\beta} & =\frac{\left(x_{1}+x_{2} \beta_{1}\right)\left(-\beta_{1}+q-b\right)}{a} \\
& =\frac{x_{2}\left(\beta_{1}(b-2 q)+q^{2}-q b+a\right)+\beta_{1}\left(x_{2}(q-b)-x_{1}\right)+x_{1}(q-b)}{a} \\
& =\frac{x_{1}(q-b)+x_{2}\left(q^{2}-q b+a\right)+\left(-x_{1}-x_{2} q\right) \beta_{1}}{a} .
\end{aligned}
$$

We define the integer division of $x$ by $\beta$ by the composition of the division above with the integer part function.

Definition 8. Let $x=x_{1}+x_{2} \beta_{1}$, the quotient of the integer division of $x$ by $\beta$, noted $\left\lfloor\frac{x}{\beta}\right\rfloor$, is defined by $\left\lfloor\frac{x}{\beta}\right\rfloor=\left\lfloor\frac{x_{1}(q-b)+x_{2}\left(q^{2}-q b+a\right)}{a}\right\rfloor+\left\lfloor\frac{\left(-x_{1}-x_{2} q\right)}{a}\right\rfloor \beta_{1}$.

Proposition 4. Let $x=x_{1}+x_{2} \beta_{1}$, and $y=y_{1}+y_{2} \beta_{1}=\left\lfloor\frac{x}{\beta}\right\rfloor$. The point $\left(y_{1}, y_{2}\right)$ is then the image of the point $\left(x_{1}, x_{2}\right)$ by the $Q L T G_{\beta_{1}}$ defined on $\mathbb{Z}^{2}$ by:

$$
\frac{1}{a}\left(\begin{array}{rr}
q-b & a-q b+q^{2} \\
-1 & -q
\end{array}\right) .
$$


A digit of the numeration system is an element $x$ of $\mathbb{Z}[\beta]$ such that $\left\lfloor\frac{x}{\beta}\right\rfloor=0$, therefore the set of digits is

$$
\mathcal{D}=\left\{x \in \mathbb{Z}[\beta] \mid\left\lfloor\frac{x}{\beta}\right\rfloor=0\right\}=\left\{x=x_{1}+x_{2} \beta_{1} \mid G_{\beta_{1}}\left(x_{1}, x_{2}\right) \in P_{O}\right\}
$$

where $P_{O}$ is the tile of order one associated to the QLT $G_{\beta_{1}}$.

Theorem 3. Let $\beta$ be an algebraic integer such that $\beta^{2}+b \beta+a=0$ and $\mathcal{D}$ the set of elements $c$ of $\mathbb{Z}[\beta]$ such that $\left\lfloor\frac{c}{\beta}\right\rfloor=0$, the three following properties are then equivalent:

1. There exists $q$ such that $(\beta, \mathcal{D})$ is a numeration system,

2. There exists $q$ such that the $Q L T G_{\beta_{1}}$ is a consistent $Q L T$,

3. $(b \geq 2)$ or $(b=1$ and $a \geq 2)$

Proof. $(\beta, \mathcal{D})$ is a numeration system if and only if for all $c \in \mathbb{Z}[\beta]$, there exist $c_{0}, c_{1}, \ldots, c_{p} \in \mathcal{D}$ such that $c=c_{0}+c_{1} \beta+\ldots+c_{p} \beta^{p}$. Moreover, this decomposition has to be unique. It is easy to see that if this decomposition exists then $c_{i}=$ $x_{i}+y_{i} \beta_{1}$ with $\left(x_{i}, y_{i}\right)=G_{\beta_{1}}^{i}(x, y)$ where $x+y \beta_{1}=c$. We conclude that the decomposition exists and is unique if and only if for each $(x, y) \in \mathbb{Z}^{2}$ there exists $p \in \mathbb{N}$ such that $G_{\beta_{1}}^{p}(x, y)=(0,0)$. Finally $(\beta, \mathcal{D})$ is a numeration system if and only if $G_{\beta_{1}}$ is a consistent QLT. Theorem 1 gives the necessary and sufficient conditions such that $G_{\beta_{1}}$ is a consistent QLT but only for QLTs such that $\left\|G_{\beta_{1}}\right\|_{\infty}<1$. The first and the third case of this theorem can not be satisfied. Indeed, in these two cases we have the condition $q^{2}-q b+a \leq 0$ and we have assumed that $b^{2}-4 a<0$ so that $q^{2}-q b+a$ is always positive. The second case corresponds to the conditions

$$
q-b \leq 0, \quad q^{2}-q b+a>0, \quad-1 \leq 0 \text { and }-1-q \leq 0
$$

which is equivalent to $-1 \leq q \leq b$. But $\left\|G_{\beta_{1}}\right\|_{\infty}<1$, implies

$\left\|G_{\beta_{1}}\right\|_{\infty}=\frac{1}{a} \max \left(b-q+q^{2}-q b+a ; 1+|q|\right)<1 \Leftrightarrow\left\{\begin{array}{r}q^{2}-(b+1) q+b<0 \\ 1+|q|<a\end{array}\right.$

If $b \leq 2$, the conditions $-1 \leq q \leq b$ and $q^{2}-(b+1) q+b<0$ are conflicting. But if $b>2$, the condition (1) is satisfied for $1<q<b$ and the condition (2) $1+q<a$ can always be satisfied (because $b^{2}<4 a$ ).

When $b=2$ the QLT associated with the integer division is of norm 1. If we choose $q=2$ the QLT is defined by the matrix $\frac{1}{a}\left(\begin{array}{rr}0 & a-1 \\ -1 & -1\end{array}\right)$ which is a consistent QLT (see [7, [1]).

When $b=1$, the QLT associated with the integer division is defined by $\frac{1}{a}\left(\begin{array}{cr}q-1 & a-q+q^{2} \\ -1 & -q\end{array}\right)$. If $a=1$ and $q>1$ the QLT is not contracting, if $a=1$ and $q=0$ or 1 the QLT is not consistent (see 7]). If $a>1$, we can choose $q=1$, then the QLT is defined by $\frac{1}{a}\left(\begin{array}{rr}0 & a \\ -1 & -1\end{array}\right)$ which is a consistent QLT. 
Example 2. Let $\beta^{2}+3 \beta+3=0$, and $\beta_{1}=1+\beta$, the QLT corresponding to the integer division by $\beta$ is defined by $\frac{1}{3}\left(\begin{array}{rr}-2 & 1 \\ -1 & -1\end{array}\right)$, the set of digits is $\mathcal{D}=$ $\{0,-1,-2-\beta\}$. If we choose $\beta_{1}=2+\beta$, the QLT corresponding to the integer division by $\beta$ is defined by $\frac{1}{3}\left(\begin{array}{rr}-1 & 1 \\ -1 & -2\end{array}\right)$, the set of digits is $\mathcal{D}=\{0,-1,-2\}$.

\section{Quasi-Linear Transformations and Fractals}

\subsection{Border of Tiles in 2-Dimension}

Let $\beta$ denote a complex number and $\mathcal{D}$ a finite set of elements of $\mathbb{Z}[\beta]$. $(\beta, \mathcal{D})$ is a valid base for $\mathbb{Z}[\beta]$ if each element $c$ of $\mathbb{Z}[\beta]$ can be written uniquely in the form $c=c_{0}+c_{1} \beta+c_{2} \beta^{2} \ldots+c_{n} \beta^{n}$ with $c_{i} \in \mathcal{D}$ and $n \in \mathbb{N}$.

Gilbert 6] proved that if $(\beta, \mathcal{D})$ is a valid base for $\mathbb{Z}[\beta]$, then every complex number $c \in \mathbb{C}$ has an infinite representation (not necessarily unique) in the form:

$$
c=\sum_{j=-\infty}^{p} c_{j} \beta^{j}, c_{j} \in \mathcal{D}
$$

Let us consider the set of complex numbers with zero integer part in this numeration system (also called "fundamental domain" in the literature [12]), that is to say the set $\mathcal{K}$ of complex numbers $c$ such that:

$$
c=\sum_{j=-\infty}^{-1} c_{j} \beta^{j}, c_{j} \in \mathcal{D}
$$

In [5] Gilbert determined the fractal dimension of the border of this set considering the base $\beta=-n+i$ with $n \in \mathbb{N}$ and $\mathcal{D}=0,1, \ldots, n^{2}$. Note $K_{p}=$ $\left\{c \in \mathbb{C} \mid c=\sum_{j=1}^{p} \frac{c_{j}}{\beta^{j}}\right\}$, the set $\mathcal{K}$ is the limit of $K_{p}$ when $p$ tends toward infinity.

Denote by $\rho$ and $\theta$ the module and argument of $\beta$ : $\beta=\rho e^{i \theta}$. We have $\rho=\sqrt{\delta}$ where $\delta$ is the determinant of the matrix associated to the division, and so:

$$
\begin{aligned}
K_{p} & =\left\{c \in \mathbb{C} \mid c=\sum_{j=1}^{p} \frac{c_{j}}{\beta^{j}} \text { with } c_{j} \in \mathcal{D}\right\} \\
& =\left\{c \in \mathbb{C} \mid c=\frac{1}{\beta^{p}} \sum_{j=1}^{p} c_{j} \beta^{p-j} \text { with } c_{j} \in \mathcal{D}\right\} \\
& =\frac{1}{\beta^{p}}\left\{c \in \mathbb{C} \mid c=\sum_{j=0}^{p-1} c_{j} \beta^{j} \text { with } c_{j} \in \mathcal{D}\right\}
\end{aligned}
$$

If $\beta$ is a Gaussian integer $\mathcal{D}=P_{O}$, if $\beta$ is an algebraic integer

$$
\mathcal{D}=\left\{x=x_{1}+x_{2} \beta_{1} \mid\left(x_{1}, x_{2}\right) \in P_{O}\right\} .
$$


In [8] we studied particular determinantal QLTs associated with matrices of the form $\frac{1}{a^{2}+b^{2}}\left(\begin{array}{cc}a & b \\ -b & a\end{array}\right)$ and showed how the border of the p-tiles of these QLTs can be generated by a substitution. This study has been generalized to every $2 \mathrm{D}$ determinantal QLT, so we define substitutions that generate the border of the p-tiles $P_{O}^{p}$. We don't give the method to generate the substitutions in this paper, it can be found in 11. The susbtitution associated with the QLT, determines the border of $P_{O}$. If at each step of the substitution we divide the length of these segments by $\sqrt{\delta}$, we obtain the border of a fractal set noted $\mathcal{F}$ (which corresponds exactely to the fundamental domain in case of Gaussian integers). Let denote by $N_{p}$ the number of segments of $P_{O}^{p}$, the susbtitution allows to determine $N_{p}$. Using the counting box dimension we can determine the fractal dimension of the border which is given by $d=\lim _{p \rightarrow+\infty} \frac{\log N_{p}}{\log \delta^{\frac{p}{2}}}$.

Example 3. Figure 3 represents a tile associated to the numeration system $(-1+$ $i,\{0,-1\})$, the associated set $K_{p}$ defined above tends to the fundamental domain of this numeration system, we obtain the fractal dimension $d=1,5236$ (which conforms to the result found in [5]). Figure 4 doesn't correspond to a numeration system, the fractal dimension of the set obtained is $d=1,303$.

\subsection{Fractals of $\mathbb{Z}^{n}$}

Consider the recurrence given in proposition 1 1 which allows the construction of $P_{O}^{n} \in \mathbb{Z}^{n}$. By applying this recurrence to a subset $P_{O}^{\prime}$ of $P_{O}$, that is to say that we define

$$
P_{O}^{\prime p+1}=\bigcup_{X \in P_{O}^{\prime}} \mathcal{T}_{\left(m \widehat{A}^{T}\right)^{p} X} P_{O}^{\prime p}=\bigcup_{X \in P_{O}^{\prime p}} \mathcal{T}_{\left(\widehat{m A}^{T}\right) X} P_{O}^{\prime}
$$

Proposition 5. Let denote $N_{p}$ the number of points of $P_{O}^{\prime p}$ and $N$ the number of points removed from $P_{O}$ to obtain $P_{O}^{\prime}$. We have $N_{p}=\left(m^{n} \delta^{n-1}-N\right)^{p}$.

Proof. In property 2 we proved that the number of points of $P_{O}$ equals $m^{n} \delta^{n-1}$ therefore $N_{1}=m^{n} \delta^{n-1}-N$. Suppose that $N_{p}=\left(m^{n} \delta^{n-1}-N\right)^{p}$, we have

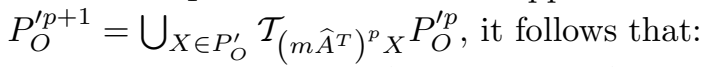

$N_{p+1}=N_{1} N_{p}=\left(m^{n} \delta^{n-1}-N\right)\left(m^{n} \delta^{n-1}-N\right)^{p}=\left(m^{n} \delta^{n-1}-N\right)^{p+1}$.

Like above, at each step of the recurrence, we divide the size of the points by $m \delta^{\frac{n-1}{n}}$. We then obtain a fractal whose box-counting dimension is given by

$$
d=\lim _{p \rightarrow \infty} \frac{\log \left(m^{n} \delta^{n-1}-N\right)^{p}}{\log \left(\left(m \delta^{\frac{n-1}{n}}\right)^{p}\right)}=\frac{\log \left(m^{n} \delta^{n-1}-N\right)}{\log \left(m \delta^{\frac{n-1}{n}}\right)}
$$

If we consider numeration systems, $P_{O}^{\prime}$ corresponds to a subset $\mathcal{D}^{\prime}$ of the set of digits $\mathcal{D}$, so that the fractal obtained corresponds to the set of numbers with zero integer part and whose decomposition uses only the digits of $\mathcal{D}^{\prime}$. 
Example 4. In figures 516 and 2 we see $P_{O}$ and the fractal generated.The black points of $P_{0}$ are removed to obtain $P_{O}^{\prime}$ (which corresponds to the grey points). The QLT in figure 5 is defined by $\frac{1}{9}\left(\begin{array}{rr}0 & -3 \\ 3 & 0\end{array}\right)$, the fractal dimension of the set is $\frac{\log (7)}{\log (3)}=1,7712$, it corresponds to the numeration system $(-3 i,\{u+i v \mid u=$ $0,1,2 v=0,-1,-2\})$ and represents the set of numbers with zero integer part and whose decomposition don't use the digits 0 and $2-2 i$. In figure 6 the QLT is defined by $\frac{1}{13}\left(\begin{array}{rr}-2 & 3 \\ -3 & -2\end{array}\right)$, the fractal dimension of the set is $2 \frac{\log (8)}{\log (13)}=1,6214$, it corresponds to the numeration system $(-2+3 i,\{0,-1,-2,-3,-4,-1+i,-2+$ $i,-3+i-4+i,-2+2 i,-3+2 I,-2-i,-3-i\})$ and represents the set of numbers with zero integer part and whose decomposition don't use the digits $0,-4,-1+i,-3+2 i$ and $-2-2 i$. In figure 2 the QLT is defined by $\frac{1}{9}\left(\begin{array}{rr}2 & 3 \\ -2 & 1\end{array}\right)$ and the fractal dimension of the set is $\frac{2 \log (5)}{\log (8)}=1,5479$.

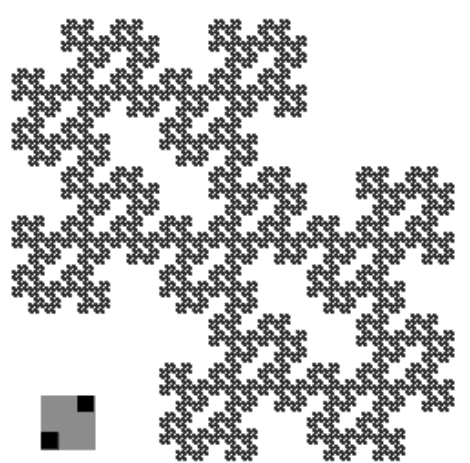

Fig. 5. Fractal associated with numeration systems

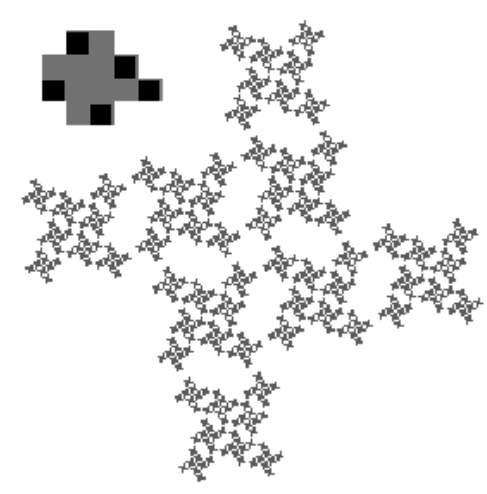

Fig. 6. Another fractal associated with numeration systems

\section{Conclusion}

We have seen relations that exist between QLTs, numeration systems and fractals. We used consistent 2D-QLTs (see definition 6) to define new numeration systems of $\mathbb{Z}[\beta]$ where $\beta$ is an algebraic integer of order 2 . These numeration systems are associated with $2 \mathrm{D}$-fractals in two ways. If we consider the set of numbers with zero integer part in the numeration system we obtain a set with fractal border. The fractal dimension of this border is determined with help of substitution associated to particular QLTs. This way to obtain fractals is generalised to all m-determinantal 2D QLTs (see definition 5). As a future work, it would be interesting to generalise this method to $\mathbb{Z}^{n}$ which implies the generalisation of substitutions in higher dimension. If we consider the set of numbers with zero integer part and whose decompositions use a subset of the set of digits 
we obtain another fractal set. Properties of the QLTs associated to the numeration systems allow to determine the fractal dimension of these sets. This way to obtain fractal (and their fractal dimension) is generalised to all m-determinantal QLTs in $\mathbb{Z}^{n}$. As a future work we would like to generalise the numeration systems to algebaic number of higher order. In [2], the author studied fractals associated with shift radix systems (which generalize numeration systems). Some of these fractals can be obtained usign QLTs, one of our future works is to study the relations between QLTs and these numeration systems.

\section{References}

1. Berthé, V., Siegel, A.: Tilings associated with Beta-Numeration and Substitutions. Integers: Electronic Journal of Combinatorial Number Theory 5 (2005)

2. Berthé, V., Siegel, A., Steiner, W., Surer, P., Thuswaldner, J.M.: Fractal tiles associated with shift radix systems. Adv. Math. (2010) (in press)

3. Blot, V., Coeurjolly, D.: Quasi-Affine Transformation in Higher Dimension. In: Brlek, S., Reutenauer, C., Provençal, X. (eds.) DGCI 2009. LNCS, vol. 5810, pp. 493-504. Springer, Heidelberg (2009)

4. Coeurjolly, D., Blot, V., Jacob-Da Col, M.-A.: Quasi-Affine Transformation in 3D: Theory and Algorithms. In: Wiederhold, P., Barneva, R.P. (eds.) IWCIA 2009. LNCS, vol. 5852, pp. 68-81. Springer, Heidelberg (2009)

5. Gilbert, W.J.: The Fractal Dimension of Sets derived from Complex Bases. Canad. Math. Bull. (1986)

6. Gilbert, W.J.: Complex Based Number Systems. University of Waterloo (2002), http://www.math.uwaterloo.ca/ wgilbert/Research/FractalPapers.html

7. Jacob, M.-A.: Applications quasi-affines. PhD thesis, Université Louis Pasteur, Strasbourg (1993)

8. Jacob, M.-A., Reveilles, J.-P.: Gaussian numeration systems. 5ème colloque DGCI (1995), https://dpt-info.u-strasbg.fr/ jacob

9. Jacob-Da Col, M.-A.: Applications quasi-affines et pavages du plan discret. Theoretical Computer Science 259(1-2), 245-269 (2001), Available in English at https://dpt-info.u-strasbg.fr/ jacob

10. Jacob-Da Col, M.-A., Tellier, P.: Quasi-linear transformations and discrete tilings. Theoretical Computer Science 410, 2126-2134 (2009)

11. Jacob-Da Col, M.-A., Tellier, P.: Quasi-linear transformations, substitutions, numerations systems and fractals. Technical Report, Laboratoire LSIIT (2010), https://dpt-info.u-strasbg.fr/ jacob

12. Kátai, I.: Generalized Number Systems in Euclidian Spaces. Mathematical and Computer Modelling 38, 883-892 (2003)

13. Klette, R., Rosenfeld, A.: Digital straightness-a review. Discrete Appleid Mathematics 139, 197-230 (2004)

14. Nehlig, P., Reveilles, J.-P.: Fractals and Quasi-Affine Transformations. Computer Grapics Forum 14, 147-158 (1995)

15. Nehlig, P.: Applications quasi-affines: pavages par images réciproques. Theoretical Computer Science 156, 1-38 (1995)

16. Nehlig, P.: Applications Affines Discrètes et Antialiassage. PhD thesis, Université Louis Pasteur, Strasbourg (1992)

17. Reveilles, J.-P.: Géométrie discrète, calcul en nombres entiers et algorithmique. Thèse d'Etat, Université Louis Pasteur, Strasbourg (1991) 